\title{
Rheumatoid arthritis-interstitial lung disease: manifestations and current concepts in pathogenesis and management
}

\author{
Suha Kadura (1) and Ganesh Raghu \\ Dept of Medicine, Center for Interstitial Lung Diseases, University of Washington, Seattle, WA, USA.
}

Corresponding author: Ganesh Raghu (graghu@uw.edu)

Shareable abstract (@ERSpublications)

Rheumatoid arthritis (RA) is a systemic inflammatory disorder, with the most common extraarticular manifestation of RA being lung involvement. RA-ILD is a leading cause of death in RA patients and is associated with significant morbidity and mortality. https://bit.ly/3w6oY4i

Cite this article as: Kadura S, Raghu G. Rheumatoid arthritis-interstitial lung disease: manifestations and current concepts in pathogenesis and management. Eur Respir Rev 2021; 30: 210011 [DOI: 10.1183/16000617.0011-2021].

\section{Abstract}

Copyright $\odot$ The authors 2021

This version is distributed under the terms of the Creative Commons Attribution NonCommercial Licence 4.0. For commercial reproduction rights and permissions contact permissions@ersnet.org

Received: 11 Jan 2021 Accepted: 13 May 2021



Pulmonary involvement in RA

Prior to utilisation of computed tomography (CT), RA-related pleural effusion was considered the most common manifestation of RA lung disease. As methodology and sensitivities for diagnostic testing have evolved over time, an increasing recognition of subtle pulmonary findings that are better identified on high-resolution CT images such as interstitial lung abnormalities and radiographic manifestations of airway 
disease (i.e. centrilobular nodules, mosaic attenuation) have led to greater identification of subclinical disease in patients with RA. Although there is extensive heterogeneity among prevalence studies of lung manifestations in RA depending on criteria for diagnosis, as well as the method and frequency of radiographic imaging, ILD has the greatest estimated prevalence, followed by airway disease, pleural effusion and rheumatoid nodules (table 1) [5-11]. The majority of respiratory manifestations occur within the first 5 years of disease, although respiratory symptoms may precede onset of articular symptoms in $10-20 \%$ of cases $[22,23]$.

\section{Predisposing factors for development of RA and associated lung disease}

\section{Genetic factors}

The genetic contribution to RA has been explored in twin studies, with previous findings demonstrating concordance rates of 15-30\% in monozygotic twins and 4\% in dizygotic twins [24, 25]. Genetic factors, mainly in the class II major histocompatibility complex (MHC) region, confer a risk of up to 50\% for the development of RA [26]. The human leukocyte antigen (HLA)-DRB1 allele, which is involved in MHC molecule-based antigen presentation and is responsible for self-peptide selection and T-cell repertoire, is the most significant genetic risk factor found to date and has been confirmed in patients who are positive for rheumatoid factor (RF) or anti-citrullinated protein antibody (ACPA) [24, 27]. In addition, a conserved amino acid sequence in the HLA-DRB chain, referred to as the shared epitope, is shared between RA-associated HLA-DR alleles and is highly associated with the presence of anti-cyclic citrullinated peptide antibodies (anti-CCP) and the development of RA [28].

\section{TABLE 1 Prevalence and clinical features of rheumatoid arthritis (RA)-associated lung diseases}

\begin{tabular}{|c|c|c|}
\hline \multicolumn{3}{|l|}{ Parenchymal lung disease } \\
\hline UIP pattern & $\begin{array}{c}\text { Radiographic pattern: subpleural, basal predominant reticular opacities, } \\
\text { honeycombing, minimal ground-glass opacity, architectural distortion with } \\
\text { traction bronchiectasis } \\
\text { Associated with worse outcomes compared to other disease } \\
\text { patterns in RA }\end{array}$ & $8-66 \%$ \\
\hline NSIP pattern & $\begin{array}{c}\text { Extensive ground-glass opacity, traction bronchiectasis, subpleural sparing } \\
\text { Lower risk of disease progression and better treatment response } \\
\text { compared with UIP }\end{array}$ & $19-57 \%$ \\
\hline Organising pneumonia & Focal ground-glass opacities, consolidations, reversed halo sign & $0-11 \%$ \\
\hline Other (e.g. LIP or DIP) & $\begin{array}{l}\text { Thin-walled cysts, centrilobular nodules, ground-glass attenuation, } \\
\text { peribronchovascular septal thickening, upper-lobe predominant }\end{array}$ & Rare \\
\hline Rheumatoid nodules & $\begin{array}{c}\text { May be single or multiple and range in size, typically asymptomatic unless } \\
\text { they cavitate or rupture, in which case infection, pleural effusion or } \\
\text { bronchopleural fistula may occur }\end{array}$ & $\begin{array}{c}<1 \% \text { radiographically, } 30 \% \text { on autopsy } \\
\text { specimens }\end{array}$ \\
\hline Caplan's syndrome & $\begin{array}{c}\text { Complication that occurs in those with pneumoconiosis from occupational } \\
\text { exposure to coal, silica or asbestos } \\
\text { Sudden development of multiple nodules, from } 0.5 \text { to several centimetres } \\
\text { in diameter, distributed throughout the lungs but predominantly at the } \\
\text { lung periphery }\end{array}$ & $<1 \%$ in the USA by autopsy \\
\hline \multicolumn{3}{|l|}{ Airway disease } \\
\hline \multicolumn{3}{|l|}{ Upper airway } \\
\hline Cricoarytenoiditis & $\begin{array}{l}\text { Arthritis of the cricoarytenoid joint leading to mid-line adduction of vocal } \\
\text { cords with resultant hoarseness and/or stridor }\end{array}$ & $\begin{array}{l}32-75 \% \text { via laryngoscopy } \\
54-72 \% \text { on CT scans }\end{array}$ \\
\hline \multicolumn{3}{|l|}{ Lower airway } \\
\hline Bronchiectasis & Associated with chronic infection & $\begin{array}{l}\text { Present on } \mathrm{HRCT} \text { in } \sim 30 \% \text {, though } \\
\text { many cases clinically silent }\end{array}$ \\
\hline $\begin{array}{l}\text { Bronchiolitis } \\
\text { (constrictive or follicular) }\end{array}$ & Female sex, high RF titre and long disease duration & $\begin{array}{l}\text { By pulmonary function testing or } \\
\text { radiographic pattern: } 8-30 \%\end{array}$ \\
\hline \multicolumn{3}{|l|}{ Pleural disease } \\
\hline Pleural effusion & $\begin{array}{l}\text { Middle-aged males with positive RF and rheumatoid nodules } \\
\text { Rheumatoid effusion: classically sterile, exudative fluid with low } \mathrm{pH}(<7.3) \text {, } \\
\text { glucose }<60 \text {, and elevated LDH }\end{array}$ & $\begin{array}{l}\text { Symptomatic in } 3-5 \% \text {, though present } \\
\text { in up to } 70 \% \text { in autopsy studies }\end{array}$ \\
\hline
\end{tabular}


Many genetic studies have identified variants that are associated with increased susceptibility to pulmonary fibrosis, with similarities between RA-ILD and familial idiopathic pulmonary fibrosis (IPF), as well as other fibrotic ILDs [29, 30]. The MUC5B promoter variant, which is involved in airway clearance and bacterial host defence, is the strongest genetic risk factor for IPF and is observed in at least $50 \%$ of patients with the disease [30, 31]. The MUC5B variant has been to shown to be associated with RA-ILD (particularly those with usual interstitial pneumonia (UIP) pattern), as well as fibrotic hypersensitivity pneumonitis (HP) [31, 32]. In addition, a study using whole exome sequencing found that patients with RA-ILD demonstrated an excess of mutations in genes that have been previously linked to familial pulmonary fibrosis including TERT, RTEL1, PARN and SFTPC [33].

\section{Environmental factors}

Among the investigated environmental risk factors, substantial evidence exists supporting cigarette smoking as a risk factor for development of seropositive RA, as well as RA-ILD. This relationship was first described over 30 years ago and has been reported in numerous subsequent studies [34-45]. In one study of patients with RA and $>25$ pack-year smoking history, those with smoking history were 3.1 times more likely to be positive for RF, and 2.4 times more likely to have joint erosions than patients with RA who did not smoke, suggesting that cumulative cigarette smoke exposure might influence the severity of articular manifestations in RA [34].

On a molecular level, smoking, and possibly other inhaled pro-inflammatory agents (such as silica dust), have been implicated in promoting protein citrullination in the lungs, a process mediated by peptidylarginine deiminase enzymes [41]. These findings suggest that smoking can modify self-proteins in the lung to become autoimmune targets [46]. Furthermore, in a large case-control study from Sweden evaluating patients with ACPA and RA, the combination of smoking history and the presence of double copies of HLA-DR shared epitope genes increased the risk for RA 21-fold compared with the risk among nonsmokers carrying no shared epitope genes, again indicating that in the context of HLA-DR shared epitope genes, smoking may trigger RA-specific immune reactions to citrullinated proteins [42].

RA-ILD

\section{Epidemiology and risk factors}

ILD is the most common pulmonary manifestation of RA lung disease, being detected in up to $60 \%$ of patients with RA on high-resolution computed tomography (HRCT), clinically significant in 10\% of cases, and is a leading cause of illness and death in patients with RA [5, 6, 8, 31]. Although RA is more common in females, RA-ILD occurs more frequently in males, with a male-to-female ratio as high as 2:1 in some studies $[47,48]$. Onset of lung disease typically occurs in the fifth to sixth decade of life. Additional risk factors associated with RA-ILD that have been reproduced across studies include smoking history, seropositivity for RF or anti-CCP antibodies, as well as RA disease activity [6, 12, 49-52].

Few genetic associations between RA and pulmonary fibrosis have been identified so far; most associations involve the MHC gene loci on chromosome 6, which has a major function of antigen presentation to T-cells at the start of an adaptive immune response.

HLA-B54, HLA-DQ1B*0601, HLA-B40, and the site encoding a-1 protease inhibitor are associated with an increased risk of ILD in patients with RA [46].

\section{Diagnosis of RA-ILD}

The diagnostic approach to patients with ILD in the setting of known or suspected RA requires a collaborative multidisciplinary approach with expert radiology, pathology, rheumatology and pulmonology input, evaluating for other potential causes of ILD such as HP, pneumoconiosis, connective tissue diseases (CTD) other than RA, or iatrogenic causes such as drug toxicity (figure 1).

\section{Imaging}

Patients with RA manifesting pulmonary symptoms need to be evaluated with chest imaging. While routine chest radiographs may be considered as a first initial step, HRCT images are essential to detect patterns and distributions of interstitial pneumonia, airway, pleural and other parenchymal abnormalities including nodules, bronchiectasis, and vascular abnormalities at baseline and to monitor disease progression over time.

While all patterns of interstitial pneumonia are known to occur in RA-ILD, the most common manifestation of RA-ILD is the UIP pattern. In a study of patients with RA-ILD, four major HRCT patterns of disease were identified, namely UIP (37\%), nonspecific interstitial pneumonia (NSIP) (30\%), obliterative bronchiolitis (17\%), and organising pneumonia (OP) (8\%) [53]. The UIP pattern is 


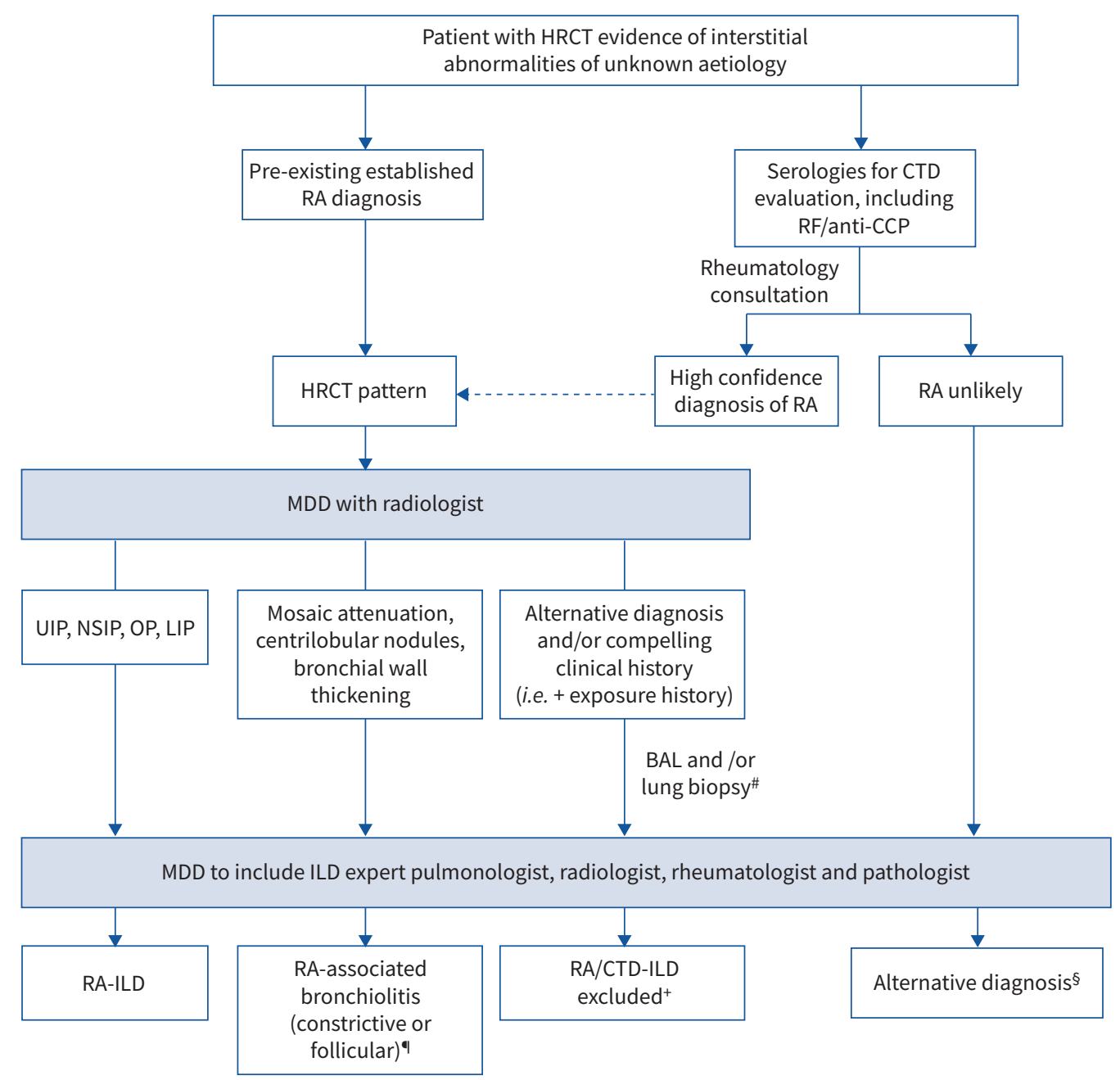

FIGURE 1 Suggested approach to the diagnosis of rheumatoid arthritis (RA) (and/or connective tissue disease (CTD))-associated interstitial lung disease (ILD). HRCT: high-resolution computed tomography; RF: rheumatoid factor; CCP: cyclic citrullinated protein; MDD: multidisciplinary discussion; UIP: usual interstitial pneumonia; NSIP: nonspecific interstitial pneumonia; OP: organising pneumonia; LIP: lymphocytic interstitial pneumonia; BAL: bronchoalveolar lavage. \#: transbronchial biopsy, cryobiopsy or surgical lung biopsy, as per the treating clinician's discretion and/or MDD; elective surgical lung biopsy in patients may be considered if transbronchial lung biopsy and/or cryobiopsy is non-diagnostic in patients who are stable and are not at high risk for surgical complications; ": findings can overlap with other radiographic patterns; ${ }^{+}$: patients should be re-evaluated when appropriate if clinical symptoms or features of CTD develop during follow-up; ${ }^{\S}$ : pursue alternative diagnoses guided by appropriate clinical setting and guidelines (e.g. idiopathic pulmonary fibrosis, hypersensitivity pneumonitis, sarcoidosis, etc.).

characterised by subpleural, basal predominant, reticular abnormalities with honeycombing, and traction bronchiectasis with a relative absence of ground-glass opacities and air trapping on exhalation (figure 2) [54]. NSIP is characterised by basilar predominant, ground-glass opacities and absence of honeycombing [55]. Additional patterns less commonly seen in RA include other patterns of interstitial pneumonia, including OP, diffuse alveolar damage, lymphocytic interstitial pneumonia (LIP) and desquamative interstitial pneumonia (DIP)-like patterns. Combined pulmonary fibrosis and emphysema (CPFE) has also been demonstrated on HRCT scans in patients with RA. HRCT image analysis in patients with smoking history with either IPF or RA-ILD revealed the coexistence of radiographically evident emphysema in close to 50\% of individuals with RA-ILD and 35\% of those with IPF, and association to lower pack-year smoking histories than in control groups [56]. These findings again provide support for possible pathogenetic linkage to smoking in both diseases. 

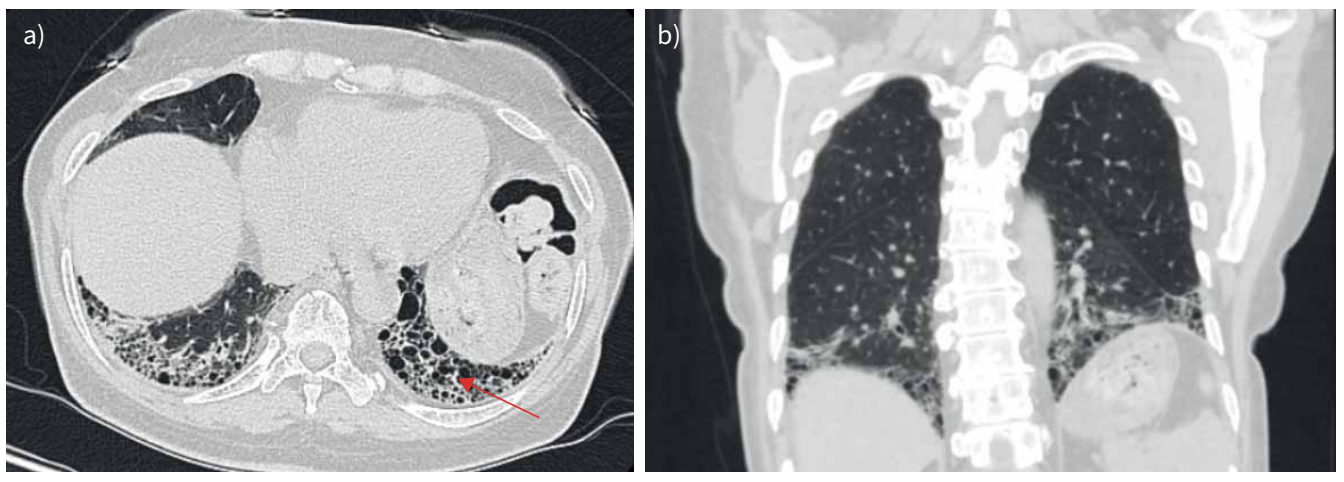

FIGURE 2 a) Axial and b) coronal computed tomography scans of usual interstitial pneumonia pattern in a 66-year-old Puerto Rican female known to have rheumatoid arthritis. Subpleural and basilar predominant reticulations, minimal ground-glass opacities, and honeycombing (arrow) are visible, as well as traction bronchiectasis.

\section{Bronchoalveolar lavage}

The cellular findings of bronchoalveolar lavage (BAL) fluid in patients with RA-ILD are often abnormal but nonspecific. Based on limited data, lymphocytosis appears to be more common in patients with RA-ILD, other than UIP pattern while increased neutrophil count is more common in patients with UIP pattern [57]. Given the nonspecific BAL findings, BAL is typically performed in the setting of clinical decompensation to rule out infection and is generally not used as a diagnostic tool.

\section{Pathology}

Although knowledge of the histopathological pattern of interstitial pneumonia by surgical lung biopsy (SLB) may clarify the diagnosis in patients manifesting imaging patterns of interstitial pneumonia (other than UIP) and could be of prognostic value, one should carefully outweigh the benefits and risks of performing SLB, as this knowledge is usually not essential to determining a treatment regimen, which generally includes immunomodulating agents [55]. It is clear that patients with definitive UIP pattern do not require SLB for the purposes of histopathology confirmation, as the correlation with radiographic UIP pattern has been well documented $[23,58,59]$. SLB can be considered in cases with HRCT patterns other than UIP where the diagnosis of CTD is not clearly established (such as in a case where lung symptoms precede articular RA manifestations), or when alternative diagnoses are being considered (figure 1) [60].

When SLB is obtained, the histopathology may reveal recognised features of UIP, NSIP, OP, DIP or LIP, and superimposed features of diffuse alveolar damage if the SLB is obtained during an acute exacerbation [55]. The histopathologic pattern of UIP may be seen in up to $61 \%$ of patients with RA-ILD [61, 62].

Subtle histopathological differences may be seen between RA-UIP and IPF. In RA-UIP, there are often fewer fibroblast foci and a higher number of germinal centres [9, 63]. In addition, lung biopsy specimens from patients with RA and UIP pattern may have an increased number of CD4+ lymphocytes compared with those from patients with IPF [64]. Of note, RA-related UIP may have concurrent presence of follicular bronchiolitis, rheumatoid nodules, or chronic pleuritis [28].

\section{Similarities of RA-ILD to IPF and other fibrotic ILDS}

RA-ILD shares several characteristics with IPF, including common environmental risk factors, a high prevalence of a UIP pattern, risk for progressive disease, and poor survival [12, 13, 31, 59, 65, 66].

Strong mechanistic similarities between RA-UIP and idiopathic forms of UIP suggest possible shared pathways of pathogenesis and mechanisms of fibrosis, opening up opportunities for development of therapeutic strategies that could improve clinical outcomes in both conditions [67]. The predominance of the UIP pattern in RA-ILD contrasts with that of other CTDs such as systemic sclerosis (SSc), where the pathologic pattern found on lung biopsies is most commonly NSIP [56]. NSIP occurs in approximately one-third of patients with RA-ILD and is associated with a longer duration of articular manifestations, lower risk of disease progression, and better treatment response compared with UIP [61, 68]. Similar to IPF, the 
clinical course of RA-ILD with UIP pattern is highly variable, and episodes of acute exacerbation can occur $[28,69]$. In addition, the UIP pattern observed in RA patients predicts worse survival (3.2 versus 6.6 years) when compared with a non-UIP pattern of RA-ILD and a similar survival to those with IPF [59, 66, 68, 70].

Pathogenesis of RA-ILD: current concepts

The pathophysiology of lung involvement in RA is a consequence of a complex relationship between genetic, environmental and autoimmune factors that contribute to the aberrant tissue response in the alveolar wall and pulmonary parenchyma, which include airways and alveolar epithelial cells, lung fibroblasts and components of extracellular matrix (figure 3).

\section{Immunopathogenesis of RA-ILD and shared mechanisms with IPF}

Patients with RA typically have circulating antibodies, most notably RF and ACPAs. These autoantibodies are present in an estimated $50-80 \%$ of RA patients and are often (though not always) present in the serum for several years prior to clinical disease onset, suggesting a sequence of events in which antibodies develop in hosts with genetic and/or environmental predispositions, leading to an inflammatory response and development of clinically apparent disease [24, 73, 74]. In individuals who are genetically predisposed, citrullination, a post-translational modification marked by the conversion of arginine to citrulline, can trigger an immune response leading to the production of ACPAs, which are strongly associated with development of RA [75, 76]. RF, an autoantibody directed against the Fc part of human immunoglobulin (Ig)G, was the first autoantibody to be described in RA. Clinically, the diagnostic utility of RF itself is somewhat limited by its relatively poor specificity, as increased RF levels can be encountered in the serum of 5-10\% of healthy patients, other CTDs, as well as various inflammatory conditions and increasing age. Higher titres of RF (i.e. three times the upper limit of normal) have greater specificity for RA. ACPAs have a similar sensitivity to RF for RA but have a much greater specificity (95-98\%) [77-79]. Both ACPAs and RF are included in the 2010 American College of Rheumatology classification criteria for RA [80].

Although potential mechanisms explaining the immunopathogenesis of RA lung disease have been identified, the site where the initial event in the RA pathogenetic cascade occurs is not known. One proposed theory is that RA-associated lung disease originates in the synovial tissue following an immune response against citrullinated proteins that subsequently cross-react with similar antigens in the lungs. The basis of this hypothesis comes from the fact that the majority of RA lung disease cases initially present with joint symptoms prior to lung involvement [28]. There has, however, been an increasing amount of evidence suggesting that inciting events leading to RA development may in fact occur at sites distal to the synovial joints [26]. The "mucosal origins" hypothesis suggests initiating events that precede symptomatic RA might originate at one or more mucosal sites (namely the oral, airway or gastrointestinal tract) and then transition to involve synovial joints [67, 81]. Although the hypothesis remains unproven, evidence from an increasing number of studies over the recent years have demonstrated a major role for the microbiome in RA development $[67,81,82]$. Of particular relevance to a mucosal origins hypothesis is the discovery of IgA ACPAs in sputum samples from individuals at risk of developing RA, since IgA antibodies are important in the regulation of mucosal defences [81, 83]. In another study involving patients with early untreated RA without lung involvement, the ACPA positive individuals had higher concentrations of ACPAs in BAL samples than in serum samples, and also had substantial evidence of immune activation in bronchial tissue when compared with controls. These findings reinforce the significant role of the lung compartment in the pathogenesis of ACPA-positive RA [84].

A recent study by ZHANG et al. [85] demonstrated evidence of the role that interleukin (IL)-17 plays in the pathogenesis of murine pulmonary fibrosis, as well as in both RA-ILD and IPF, which may have clinical implications for therapeutic strategies targeting pulmonary fibrosis in RA-ILD as well as IPF. TH17 cytokines, such as IL-17A and transforming growth factor- $\beta 1$, cause fibrosis through direct effects on fibroblasts, leading to their proliferation and extracellular matrix generation [85, 86]. In addition, citrullination of lung tissue has been reported in both RA-ILD as well as IPF, and is another shared mechanism in the immunopathogenesis of both conditions [83]. One study identified rates of protein citrullination in lung tissue of IPF patients (44\%) as being similar to those found in RA-ILD patients (46\%) [49]. It has been speculated that differences between these two disease entities, including extra-pulmonary manifestations, may reflect immunogenetic factors (such as the HLA-DR genes) that may ultimately lead to citrulline-target immune responses mediated by ACPAs and possible articular disease [83]. Despite shared immune mechanisms between RA-ILD and IPF, there are also important differences that exist. Lung tissue from RA-ILD patients contains greater numbers of inducible bronchial-associated lymphoid tissue than IPF patients, suggesting that immune dysregulation may play a more central role in RA-ILD than IPF [87]. 


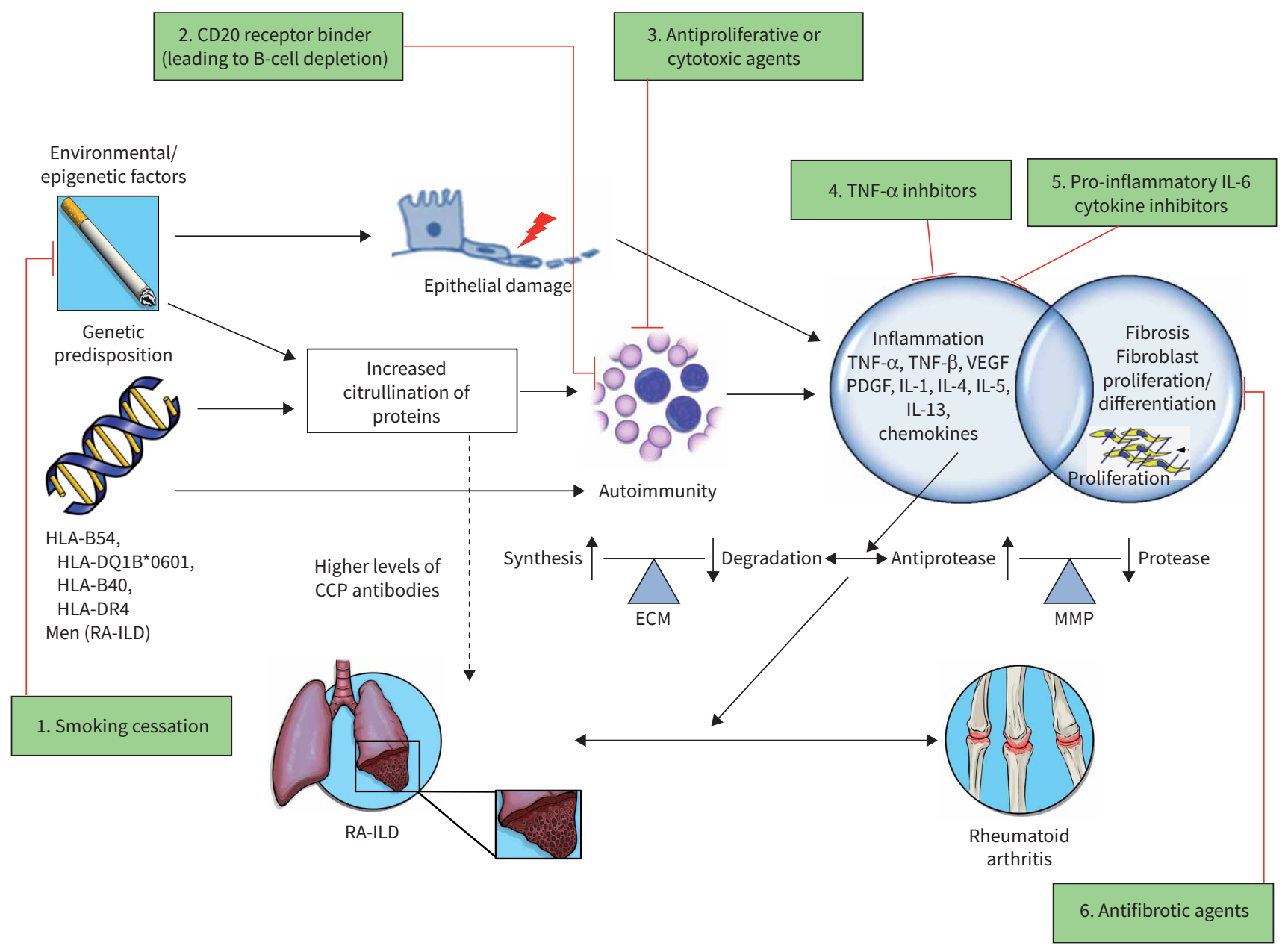

FIGURE 3 Schematic illustration of the concepts in the pathogenesis of rheumatoid arthritis (RA)-associated interstitial lung disease (ILD) with various therapeutic targets. The pathogenesis of RA is thought to involve an interplay between various risk factors (including smoking history, male sex and older age) and genetic predisposition (such as the shared epitope HLA-DRB1). In the lung, various environmental exposures causing airway and alveolar epithelial cell damage may lead to increased citrullination of proteins which, in an individual who is genetically predisposed, is thought to trigger an inflammatory process characterised by activation of cytokines, chemokines and growth factors, such as tumour necrosis factor (TNF), vascular endothelial growth factor (VEGF), platelet-derived growth factor (PDGF) and interleukins (IL). These contribute to both proliferation and differentiation of fibroblasts, increased synthesis and deposition of extracellular matrix (ECM), and increased activity of matrix metalloproteinases (MMP), resulting in the development of ILD and pulmonary fibrosis. Fibroblasts in the synovial lining play a similar role in the pathogenesis of joint manifestations of RA. Various therapeutic targets include: 1) exposure avoidance via smoking cessation; 2) binding of CD20 receptor, leading to B-cell depletion (e.g. rituximab); 3) antiproliferative or cytotoxic agents (e.g. cyclophosphamide and mycophenolate); 4) TNF- $\alpha$ inhibitors (e.g. adalimumab, etanercept and infliximab); 5) pro-inflammatory IL-6 cytokine inhibitors (e.g. tocilizumab); and 6) antifibrotic agents (nintedanib and pirfenidone). Therapeutic agents not included in the figure include corticosteroids, which promote an anti-inflammatory response via inhibition of prostaglandin and leukotriene synthesis, and reduction of the number of circulating monocytes, as well as inhibiting the release of collagenase and lysosomal enzymes [71]. Also not pictured are nonbiologic (i.e. traditional or conventional) disease-modifying anti-rheumatic drugs, such as methotrexate, for which the exact mechanism in RA is unknown, but is thought to involve adenosine signalling via increase of adenosine levels leading to an intracellular cascade promoting an overall anti-inflammatory state [72]. CCP: cyclic citrullinated peptide; HLA: human leukocyte antigen. Reproduced and modified from [60] with permission.

Understanding RA-ILD pathogenesis via animal models

Acknowledging that there are currently no widely accepted animal models for RA-ILD, the most commonly employed methods used to generate animal models in RA-ILD include induction via immune stimulants or through genetic engineering via gene mutation or transgenic systems [88]. Though there are multiple models of RA designed to replicate joint pathology, there are currently only three main arthritic models which contain lung pathology: the SKG murine model; the adjuvant arthritis model; and the tumour necrosis factor (TNF)-transgenic model [89]. 
RA-ILD animal models have led to improved understanding of pulmonary pathology secondary to systemic inflammation [89]. There are, however, limitations to RA-ILD animal models, as they cannot replicate clinical pathology and are limited in experimental research application. All known models of RA-ILD up to this point closely resemble cellular NSIP, with limited evidence of fibrosis and absence of UIP features, which is most frequently encountered in RA-ILD. Although speculative, UIP as a continuum of NSIP has been proposed as a possible explanation for this discrepancy [89]. Further exploration and improvement of animal models to more closely resemble clinical features of human RA-ILD is needed.

\section{Treatment}

In patients diagnosed with RA-ILD, it is important that a baseline assessment of disease severity is made and that patients are diligently followed in order to identify those who develop disease progression. The severity and progression of disease are two major factors to consider when deciding whether to initiate or augment ongoing treatment in patients with RA-ILD. These factors can be assessed clinically through subjective assessment of symptom worsening, decline in pulmonary function tests (PFTs), or radiologic deterioration. Other factors of the individual patient such as age, comorbidities, UIP or patterns other than UIP, and patient wishes should be taken into account, since there are no randomised controlled trials (RCTs) to date to facilitate discussions regarding treatment choice based on evidence.

\section{Immunomodulating agents}

Corticosteroids, cyclophosphamide and mycophenolate

The optimal treatment strategy for patients with RA-ILD has not been well studied. To date, there have been no RCTs comparing medications for the treatment of RA-ILD.

Treatment with immunosuppressive agents is generally used regardless of the pattern of fibrosis, though research is much needed to address whether this is the best strategy. Corticosteroids are the mainstay of therapy in RA-ILD, particularly for cases of NSIP or OP where they may lead to regression of consolidation on imaging and potential clinical improvement [90]. A retrospective study by SoNG et al. [91] demonstrated that in patients with RA-UIP, treatment with glucocorticoids alone or in combination with immunosuppressive medications improved or stabilised the disease in about $50 \%$ of 84 patients, but without significant difference in survival with the untreated group. In another recent retrospective study of 26 patients with CTD-ILD (including 11 patients with RA-ILD), those treated with pulse-dose steroids followed by 1 year of tacrolimus combination therapy with corticosteroids showed improvement in forced vital capacity (FVC), walk test variables, and patient-reported outcomes, including in the majority of patients with UIP pattern [92]. This is in contrast to IPF, in which the use of immunosuppressive therapy has been associated with worse outcomes and is contraindicated [93].

Cyclophosphamide (CYC) and mycophenolate mofetil (MMF) have been used with varying success, though the suggestion for use are based on limited retrospective and/or uncontrolled studies [94-99]. In a retrospective study of 21 patients with progressive RA-ILD (14 with UIP pattern), mean survival time was better in those treated with pulse CYC than in patients with better baseline lung function who did not receive CYC (72 versus 43 months) [95]. A major downside of treatment with CYC is the potential for devastating toxicity associated with its use, including haemorrhagic cystitis, bladder cancer, bone marrow suppression, increased risk of opportunistic infections, and haematological and solid organ malignancies, limiting its use as a long-term treatment [98].

While the treatment with MMF (and CYC) is extrapolated from studies in patients with SSc-ILD, there are no prospective studies examining efficacy of MMF in patients with RA-ILD [100, 101]. There have been several small studies that have demonstrated stabilisation and/or improvement in symptoms, lung function and imaging with use of MMF [96, 97]. In a review of 125 patients with various CTD-ILDs (18 with RA-ILD), treatment with MMF for a median of 897 days was associated with improvements in FVC \% predicted and diffusing capacity of the lung for carbon monoxide in patients with non-UIP pattern and led to stabilisation among those with UIP. The drug was well tolerated in general, with a discontinuation rate due to adverse events of $<10 \%$ [97].

DMARDs, biologic agents and potential for pulmonary toxicity

A challenge in treating RA-ILD lies in the fact that many of the therapeutic options for RA such as DMARDs and biologic agents have been linked to pulmonary toxicity (albeit rare). While DMARDs and biologic agents are widely used for the joint manifestations of RA, the potential treatment benefits for RA-ILD are unknown. 
The temporal relationship to onset of new pulmonary manifestations and initiation of therapy is crucial in raising the index of clinical suspicion of drug-induced lung toxicity in methotrexate (MTX) as well as biologic drugs, such as TNF-modulating agents. This can be quite challenging in patients with pre-existing ILD, though development of new parenchymal abnormalities in these patients in whom the differential diagnosis includes drug-induced lung toxicity should be evaluated further with appropriate diagnostic and therapeutic interventions (figure 4).

\section{Methotrexate}

MTX is the most common first-line agent used to treat RA that prevents joint destruction. A possible link between this medication and lung disease was first reported in 1983; since then, numerous additional cases have been reported [102-104]. Acute/subacute HP has been well-described in the literature, with a variable prevalence ranging from $1-2 \%$, with a higher dose more likely to be associated with pulmonary toxicity, typically occurring within the first year of treatment [105-107]. Most of the literature demonstrating this association comes from observational studies, which indeed have their limitations, the most relevant of which are a susceptibility to ascertainment and channelling biases [108]. More recent studies have suggested that MTX-associated HP is much less common than previously thought. Notably, since 2001, no cases have been reported in RCTs of MTX in RA [102, 109]. Furthermore, a meta-analysis of double-blind RCTs in patients with RA treated with MTX found an increased risk of infectious but not

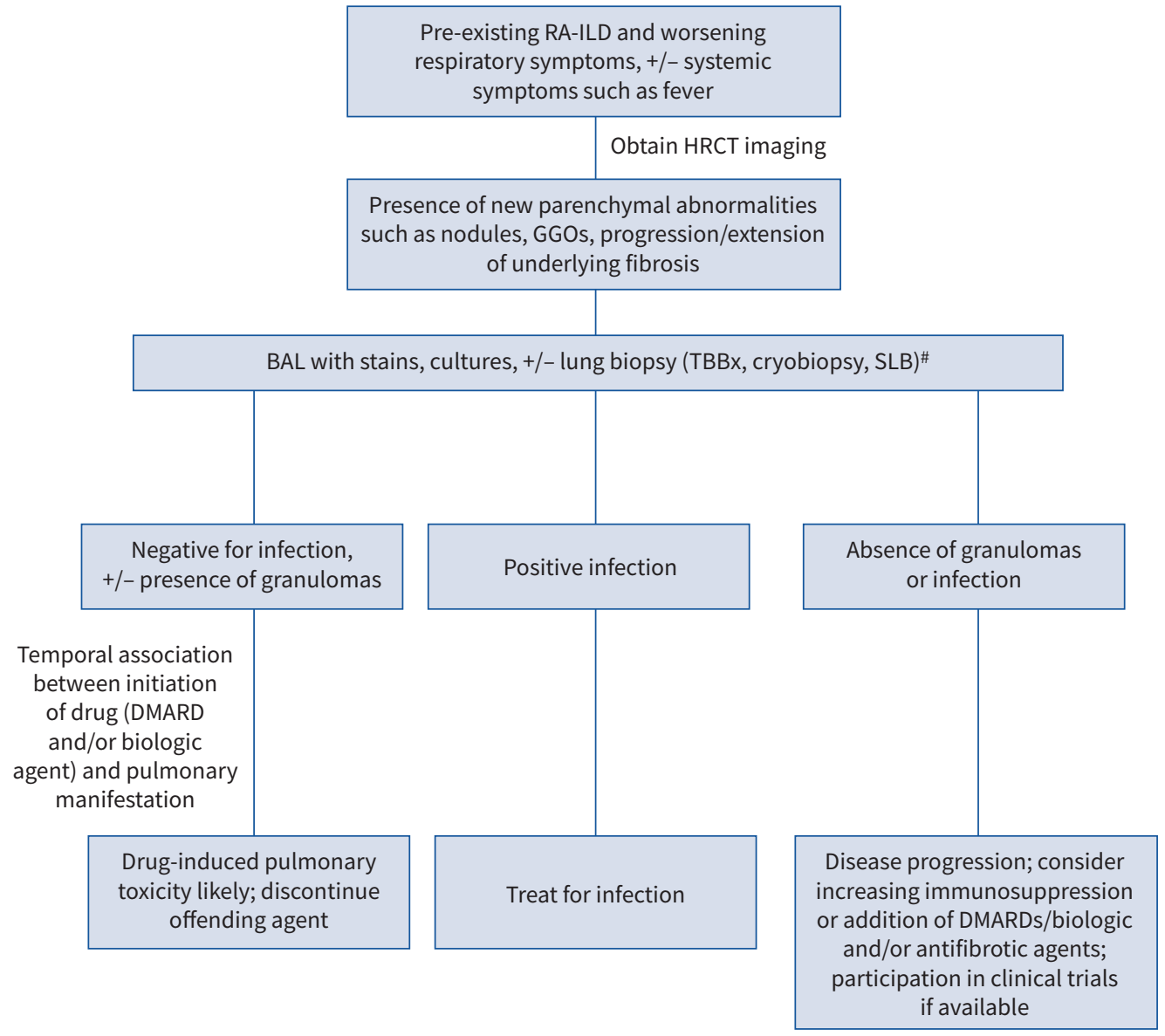

FIGURE 4 Suggested diagnostic approach for management of lung disease progression versus pulmonary infection or toxicity induced by disease-modifying anti-rheumatic drugs (DMARDs) in a patient with pre-existing rheumatoid arthritis-associated interstitial lung disease (RA-ILD). HRCT: high-resolution computed tomography; GGOs: ground-glass opacities; BAL: bronchoalveolar lavage; TBBx: transbronchial lung biopsy; SLB: surgical lung biopsy. ": transbronchial biopsy or cryobiopsy, as per the treating clinician and/or multidisciplinary discussion; elective SLB in patients may be considered if TBBx and/or cryobiopsy is non-diagnostic in patients who are stable and are not at high risk for surgical complications. 
non-infectious pulmonary disease associated with MTX use, casting further doubt on previously held notions of the drug's pulmonary effects $[102,108]$.

Nonetheless, due to the potential of pulmonary toxicity associated with MTX use, it is seldom used as an agent for treatment of RA-ILD.

Biologic drugs including anti-TNF agents

Biologic drugs are used to treat moderate-to-severe RA in patients who have not responded adequately to traditional DMARDs [110, 111]. TNF- $\alpha$ inhibitors (TNFi) may have both profibrotic and antifibrotic effects, the imbalance between these two roles may potentially trigger fibrosis or stabilise ILD [72, 112, 113].

Similar to MTX, controversy exists for both TNFi and rituximab in the treatment of ILD, with some studies showing improvement and others demonstrating development or progression of ILD [114-118]. In one study assessing 122 cases of new-onset or exacerbated ILD in the setting of TNFi use, 108 cases were patients with RA. Of note, $63 \%$ of these patients had been treated with MTX, and 38\% had pre-existing ILD [118]. In contrast, a large cohort study of 8417 patients with autoimmune disease did not show any significant difference in the incidence of ILD between those who were treated with anti-TNF therapy $(0.5 \%)$ and those were treated with other therapies $(0.3 \%)$. The incidence of ILD occurring in patients with RA was seven times higher than that for other CTDs, but again no significant difference was noted between those who received anti-TNF therapy and those who did not [117]. Though not specifically evaluating patients with RA-ILD, in a prospective randomised, double-blind clinical trial, etanercept did not demonstrate therapeutic potential and/or pulmonary toxicity in patients with IPF [119]. In a more recent prospective study in patients with RA-ILD, DETORAKIs et al. [116] found that in RA patients with or without ILD treated with TNFi, the extent of radiographic disease remained largely unchanged after 1 year of follow-up in those with RA-ILD, and none of the patients in the RA non-ILD group developed new-onset ILD. There was also no exacerbation of ILD associated with TNFi therapy [116].

\section{Antifibrotic therapy for progressive fibrotic ILD}

Given the mechanistic similarities between RA-related UIP and IPF, there is increasing interest regarding the potential benefit of antifibrotic treatment in patients with progressive fibrotic RA-ILD, particularly in those with UIP pattern. It is unknown if antifibrotic medications are effective for articular manifestations of the disease, and concomitant treatment with immunomodulating therapies may be required [113].

Results of the INBUILD trial suggest a treatment benefit with nintedanib in patients manifesting pulmonary disease progression when a heterogeneous group of patients with progressive fibrotic ILD (PF-ILD) (other than IPF) were grouped together as a single entity [120]. A sub-group, post-hoc analysis suggested a treatment benefit (specifically rate of FVC decline) across all diagnostic subgroups (including some autoimmune-ILD), although the trial was not designed or powered to provide evidence for benefit of nintedanib in specific diagnostic subgroups [121]. Additionally, it is unknown how many patients (if any) had RA-ILD in the INBUILD trial, making the benefit of nintedanib unclear in the specific cohort of patients with RA-ILD. While it is not associated with an improvement in lung function, nintedanib has been shown to reduce the decline in FVC by about $50 \%$ in the heterogeneous group of PF-ILD patients with UIP pattern, hence the concept that PF-ILD, regardless of the underlying aetiology, may be amenable to antifibrotic treatment $[30,120]$.

Pirfenidone reduces levels of IL-6 and TNF- $\alpha$, both key cytokines in RA pathogenesis [122]. It has also recently been shown to have an inhibitory effect on transition from fibroblast to myofibroblast in RA-ILD lung specimens [123]. This background may suggest a plausible rationale in the use of pirfenidone in patients with RA-ILD, especially in UIP pattern.

Data from a recent clinical trial in patients with unclassifiable PF-ILD suggested that pirfenidone may reduce disease progression [124]. In addition, results of the RELIEF trial, a phase II randomised, double-blind, placebo-controlled trial designed to evaluate safety and efficacy of pirfenidone for progressive, non-IPF lung fibrosis, are eagerly awaited [125]. While the TRAIL1 study, a phase 2, randomised, double blind, placebo-controlled trial, was intended to determine the safety and efficacy of pirfenidone in RA-ILD, the study was recently aborted for a regrettable inability to recruit the number of patients required within the planned time frame amid the coronavirus disease 2019 pandemic. Analyses of the data gathered are anticipated.

Given both the inflammatory and fibrotic mechanisms are involved in RA-ILD, combination therapy with anti-inflammatory/immune modulating and antifibrotic agents is a potential treatment strategy in some 
patients, though this will need to be explored further in prospective studies [113]. In the interim, antifibrotic therapy for non-IPF, PF-ILD may be an option for consideration in some patients as an add-on or a second-line therapy after demonstration of progressive fibrosis despite conventional treatment [126].

There are just a few studies currently evaluating the safety, tolerability and efficacy of both immunosuppressive as well as antifibrotic drugs in RA-related ILD (https://clinicaltrials.gov NCT03084419: abatacept in RA-ILD; NCT02808871: pirfenidone versus placebo; NCT04311567: tofacitinib versus methotrexate).

\section{Other treatment considerations}

Other treatment considerations not discussed in this review include cessation of cigarette smoking, pulmonary rehabilitation, oxygen supplementation, treatment of associated pulmonary hypertension as well as other comorbidities including abnormal gastro-oesophageal reflux, and lung transplant consideration when appropriate.

\section{Prognosis and predictors of mortality in RA-ILD}

Both cardiac and respiratory manifestations are leading causes of mortality in patients with RA [127, 128]. In a study by Solomon et al. [66] in patients with RA-ILD followed from 1995 to 2013 with either NSIP or UIP patterns, it was found that after controlling for potential confounding variables (such as age, sex, smoking and HRCT pattern) a lower baseline FVC \% predicted as well as a 10\% decline in FVC \% from baseline to anytime during follow-up were both independently associated with an increased risk of death, though HRCT pattern was not a significant predictor of mortality. Patients with RA-UIP had shorter survival time than those with RA-NSIP (47\% died versus 24\%, respectively) [66]. These findings put together suggest that in patients with significant baseline physiologic impairment or evidence of physiologic disease progression over time (as defined by decline in FVC \%) there is a greater risk of death, regardless of the chest computed tomography imaging pattern. A recent retrospective study that followed patients with RA and RA-ILD from 2005 to 2018 found that although overall RA-related mortality rates decreased from 30.6 per 1000000 population in 2005 to 22.2 in 2018, the RA-ILD mortality rates remained stable in both sexes, all races, and all age groups except for ages 65 to 84 years, in which the rates declined [129]. These findings suggest that improved therapies and management of RA and RA-associated comorbidities may have led to improved overall outcomes for patients with RA, but have had limited effect in the subgroup of patients with RA-ILD.

\section{Airway disease}

Prevalence of airway disease in RA is high and can occur in 39-60\% of patients [70, 130, 131]. Both the large (upper and lower) and distal small airways can be involved. The most common manifestations are bronchiectasis, bronchiolitis, airway hyperreactivity and cricoarytenoid arthritis [132].

\section{Upper airway involvement}

Upper airway disease manifestations in RA include arthritis of the cricoarytenoid joint leading to midline adduction of vocal cords with resultant hoarseness and/or stridor, vasculitis affecting the recurrent laryngeal or vagus nerves leading to vocal cord paralysis, as well as rheumatoid nodules involving the vocal cords [60, 132].

\section{Lower airway involvement}

Causes of lower airway disease in RA include bronchial hyperresponsiveness, bronchiectasis, or bronchiolitis. Bronchiectasis has been demonstrated on HRCT in 30\% of RA cases, although it may be clinically silent and can both precede or follow the development RA [7, 133]. Several hypotheses have been put forward to explain the mechanisms underlying the development of bronchiectasis in RA, including a link between autoimmunity in RA leading to airway damage, as well as chronic suppurative infections (which is perhaps enhanced in the setting of RA and/or treatment with immunomodulating medications) leading to bronchiectasis [60].

When bronchiectasis is severe enough to produce clinical symptoms, it may complicate the use of immunosuppressive medications, particularly biologic agents, as both bronchiectasis and therapy with these agents are independent risk factors for lower respiratory tract infections [134]. There is no specific therapy for patients with bronchiectasis associated with RA. Treatment of bronchiectasis in RA does not differ from either condition alone and involves bronchodilators, bronchial hygiene, and antibiotics when needed in the case of infection [133]. 
Two forms of small airway disease (follicular bronchiolitis and constrictive bronchiolitis) have been described in association with RA [132]. Follicular bronchiolitis occurs in the setting of hyperplasia of bronchial-associated lymphoid tissue and can also be seen in other CTDs. HRCT demonstrates centrilobular peribronchial nodules $<3 \mathrm{~mm}$ in size with branching structures corresponding to bronchial dilation and wall thickening (figure 5) [135]. Histopathological findings include the presence of hyperplastic lymphoid follicles with reactive germ cell centres within bronchiole walls [132]. Treatment is directed at the underlying RA, and additional treatment may not be necessary for mild disease. For more severe or symptomatic disease, corticosteroids and macrolide antibiotics have been used [135].

Constrictive bronchiolitis (or bronchiolitis obliterans) is a rare, small airways disease characterised by destruction of bronchiolar epithelium and subsequent progressive airflow obstruction [136, 137]. It is more common in females, those with positive RF, and longstanding untreated disease and has also been associated with certain medications including gold, penicillamine and sulfasalazine. Though bronchiolitis obliterans in the setting of organ transplant has been well-described, the disease course in non-transplant patients (most commonly associated with CTD) remains poorly understood [132, 137]. HRCT findings are nonspecific, but may show centrilobular nodules, bronchial wall thickening, and mosaic attenuation. PFTs generally show airflow obstruction with normal diffusing capacity of the lung for carbon monoxide.

Prognosis of RA bronchiolitis is generally thought to be poor; however, in a prospective study in 15 patients with RA and biopsy-proven follicular or constrictive bronchiolitis (in which $50 \%$ of patients had $\mathrm{RA})$, the forced expiratory volume in $1 \mathrm{~s}\left(\mathrm{FEV}_{1}\right)$ showed stability over time in both patients with and without bronchiolitis over 3 years of follow-up [132, 138]. These results should, however, be interpreted with caution, as one-third of patients in the study had history of tobacco use (which may have had an impact on $\mathrm{FEV}_{1}$ decline). Another retrospective study in patients with bronchiolitis obliterans and RA included more than half of patients with emphysema, again making interpretation of lung function testing challenging [139]. In the largest available retrospective study to date of 41 patients with RA-bronchiolitis obliterans in which those with radiologic evidence of emphysema were excluded, findings demonstrated a fairly stable clinical course despite persistent symptoms and severe airflow obstruction on presenting $\mathrm{FEV}_{1}$. All-cause mortality was $27 \%$ over a median follow-up of 62 months [137]. These findings may suggest that bronchiolitis associated with CTD may have a less aggressive course than idiopathic disease.

Given the rarity of the disease, large, randomised trials for bronchiolitis obliterans in the setting of RA are lacking. Medical therapy including a combination of long-acting inhalers, systemic corticosteroids or other immunosuppressive agents, and macrolide antibiotics are often used with unclear benefit, though a few case reports have described some improvement with anti-TNF therapy [140]. Case series have
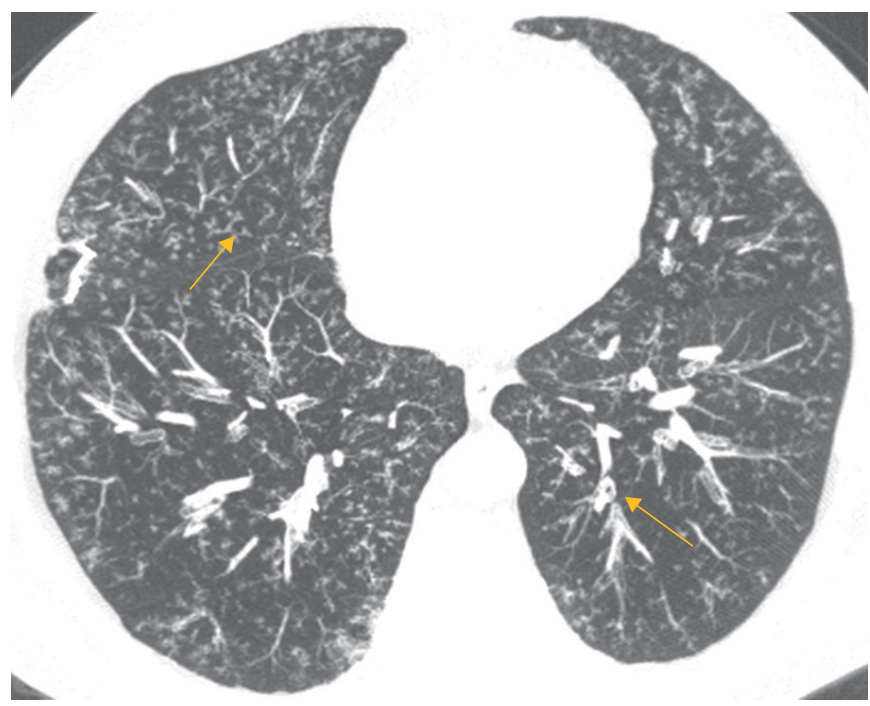

FIGURE 5 Axial computed tomography scan demonstrating classic findings of follicular bronchiolitis in a 55 -year-old male with rheumatoid arthritis. Note bilateral diffuse centrilobular peribronchial nodules $<3 \mathrm{~mm}$ in size with branching structures corresponding to bronchial dilation and wall thickening (arrows). Image courtesy of Sudhakar Pipavath (University of Washington Medical Center, Seattle, WA, USA). 
demonstrated that macrolides improve or slow the progression of respiratory impairment in RA and associated bronchiolitis obliterans, which is also evident for other bronchiolar diseases [135, 141]. In severe cases, lung transplantation may be necessary.

\section{Conclusions}

The type and prevalence of manifestations of lung disease in RA varies, and can essentially affect any lung compartment, including the lung parenchyma, pleura, airways and vasculature. Mechanisms of lung injury have been attributed to an interaction between genetic, environmental, and autoimmune factors, as well as superimposed toxicity related to medications. To date, there have been no RCTs for RA lung disease, limiting guidance for treatment other than immunomodulating agents that are used for RA in general, being aware that some of these agents are associated with pulmonary toxicity without substantial evidence of efficacy.

Over the years, improved understanding of the biology and expected disease course, as well as overlapping disease mechanisms identified in patients with RA-UIP and IPF, has led to consideration of additional treatment options for those with RA and PF-ILD, namely antifibrotic agents. Further knowledge of disease pathways in patients with RA lung disease will help to guide treatment with immunomodulating medications that will target specific pathways relevant to the disease and can perhaps be combined with antifibrotic agents when needed as a multimodal approach.

\section{Future directions}

There are several areas within the realm of RA-ILD (and more broadly CTD-ILD) for which there are continued clinical challenges and unmet research needs [142]. Future directions should include a multidisciplinary collaborative effort to both the care and research of patients with RA-ILD. Research initiatives should prioritise standardised international criteria for the diagnosis and classification of CTD-ILD (including RA-ILD), the development of strategies to allow for earlier identification of lung involvement in those with RA, as well as to predict those at highest risk for disease progression. Additionally, the identification of validated disease biomarkers is needed to aid in diagnosis, prognostication and assessment of disease progression, as well as to evaluate individual treatment response. Lastly, there remains an urgent need for the design of clinical trials for novel treatments of RA lung disease that will improve outcomes, including quality of life that are meaningful to this group of patients.

Provenance: Commissioned article, peer reviewed.

Conflict of interest: S. Kadura has nothing to disclose. G. Raghu reports personal fees and other funding from Boehringer-Ingelheim and other funding from Roche-Genentech, outside the submitted work.

\section{References}

$1 \quad$ Hunter TM, Boytsov NN, Zhang X, et al. Prevalence of rheumatoid arthritis in the United States adult population in healthcare claims databases, 2004-2014. Rheumatol Int 2017; 37: 1551-1557.

2 Myasoedova E, Crowson CS, Kremers HM, et al. Is the incidence of rheumatoid arthritis rising? Results from Olmsted County, Minnesota, 1955-2007. Arthritis Rheum 2010; 62: 1576-1582.

3 Wilsher M, Voight L, Milne D, et al. Prevalence of airway and parenchymal abnormalities in newly diagnosed rheumatoid arthritis. Respir Med 2012; 106: 1441-1446.

4 Norton S, Koduri G, Nikiphorou E, et al. A study of baseline prevalence and cumulative incidence of comorbidity and extra-articular manifestations in RA and their impact on outcome. Rheumatology (Oxford) 2013; 52: 99-110.

5 Hyldgaard C, Hilberg $\mathrm{O}$, Pedersen $\mathrm{AB}$, et al. A population-based cohort study of rheumatoid arthritis-associated interstitial lung disease: comorbidity and mortality. Ann Rheum Dis 2017; 76: 1700-1706.

6 Koduri G, Norton S, Young A, et al. Interstitial lung disease has a poor prognosis in rheumatoid arthritis: results from an inception cohort. Rheumatology (Oxford) 2010; 49: 1483-1489.

7 Perez T, Remy-Jardin M, Cortet B. Airways involvement in rheumatoid arthritis: clinical, functional, and HRCT findings. Am J Respir Crit Care Med 1998; 157: 1658-1665.

8 Olson AL, Swigris JJ, Sprunger DB, et al. Rheumatoid arthritis-interstitial lung disease-associated mortality. Am J Respir Crit Care Med 2011; 183: 372-378.

9 Brazeau-Lamontagne L, Charlin B, Levesque RY, et al. Cricoarytenoiditis: CT assessment in rheumatoid arthritis. Radiology 1986; 158: 463-466.

10 Lawry GV, Finerman ML, Hanafee WN, et al. Laryngeal involvement in rheumatoid arthritis. Arthritis Rheum 1984; 27: 873-882. 
Balbir-Gurman A, Yigla M, Nahir AM, et al. Rheumatoid pleural effusion. Semin Arthritis Rheum 2006; 35 368-378.

Kelly CA, Saravanan V, Nisar M, et al. Rheumatoid arthritis-related interstitial lung disease: associations, prognostic factors and physiological and radiological characteristics - a large multicentre UK study. Rheumatology (Oxford) 2014; 53: 1676-1682.

Kim EJ, Collard HR, King TE. Rheumatoid arthritis-associated interstitial lung disease: the relevance of histopathologic and radiographic pattern. Chest 2009; 136: 1397-1405.

Balbir-Gurman A, Guralnik L, Yigla M, et al. Imaging aspects of interstitial lung disease in patients with rheumatoid arthritis: Literature review. Autoimmun Rev 2018; 17: 87-93.

Oliveira DS, Araújo Filho JDA, Paiva AFL, et al. Idiopathic interstitial pneumonias: review of the latest American Thoracic Society/European Respiratory Society classification. Radiol Bras 2018; 51: 321-327.

Assayag D, Lee JS, King TE. Rheumatoid arthritis associated interstitial lung disease: a review. Medicina (Mex) 2014; 74: 158-165.

Duarte AC, Porter JC, Leandro MJ. The lung in a cohort of rheumatoid arthritis patients - an overview of different types of involvement and treatment. Rheumatology (Oxford) 2019; 58: 2031-2038.

Hanaka $\mathrm{T}$, Kido $\mathrm{T}$, Ishimoto $\mathrm{H}$, et al. Radiological patterns of pulmonary involvement may predict treatment response in rheumatoid arthritis: a retrospective study. Respir Investig 2019; 57: 172-182.

Salaffi F, Carotti M, Di Carlo M, et al. High-resolution computed tomography of the lung in patients with rheumatoid arthritis: prevalence of interstitial lung disease involvement and determinants of abnormalities. Medicine (Baltimore) 2019; 98: e17088.

Tanaka N, Kunihiro $\mathrm{Y}$, Kubo $\mathrm{M}$, et al. HRCT findings of collagen vascular disease-related interstitial pneumonia (CVD-IP): a comparative study among individual underlying diseases. Clin Radiol 2018; 73: 833.e1-833.e10.

Benedek TG. Rheumatoid pneumoconiosis. Documentation of onset and pathogenic considerations. Am J Med 1973; 55: 515-524.

Marigliano B, Soriano A, Margiotta D, et al. Lung involvement in connective tissue diseases: a comprehensive review and a focus on rheumatoid arthritis. Autoimmun Rev 2013; 12: 1076-1084.

O'Dwyer DN, Armstrong ME, Cooke G, et al. Rheumatoid arthritis (RA) associated interstitial lung disease (ILD). Eur J Intern Med 2013; 24: 597-603.

McInnes IB, Schett G. The pathogenesis of rheumatoid arthritis. N Engl J Med 2011; 365: 2205-2219.

MacGregor AJ, Snieder H, Rigby AS, et al. Characterizing the quantitative genetic contribution to rheumatoid arthritis using data from twins. Arthritis Rheum 2000; 43: 30-37.

Anderson R, Meyer PWA, Ally MMTM, et al. Smoking and air pollution as pro-inflammatory triggers for the development of rheumatoid arthritis. Nicotine Tob Res 2016; 18: 1556-1565.

van Drongelen V, Holoshitz J. Human leukocyte antigen-disease associations in rheumatoid arthritis. Rheum Dis Clin North Am 2017; 43: 363-376.

Spagnolo P, Lee JS, Sverzellati N, et al. The lung in rheumatoid arthritis: focus on interstitial lung disease. Arthritis Rheumatol 2018; 70: 1544-1554.

Adegunsoye A, Vij R, Noth I. Integrating genomics into management of fibrotic interstitial lung disease. Chest 2019; 155: 1026-1040.

Wijsenbeek M, Cottin V. Spectrum of fibrotic lung diseases. N Engl J Med 2020; 383: 958-968.

Juge P-A, Lee JS, Ebstein E, et al. MUC5B promoter variant and rheumatoid arthritis with interstitial lung disease. N Engl J Med 2018; 379: 2209-2219.

Ley B, Torgerson DG, Oldham JM, et al. Rare protein-altering telomere-related gene variants in patients with chronic hypersensitivity pneumonitis. Am J Respir Crit Care Med 2019; 200: 1154-1163.

Juge PA, Borie R, Kannengiesser C, et al. Shared genetic predisposition in rheumatoid arthritis-interstitial lung disease and familial pulmonary fibrosis. Eur Respir J 2017; 49: 1602314.

34 Saag KG, Cerhan JR, Kolluri S, et al. Cigarette smoking and rheumatoid arthritis severity. Ann Rheum Dis 1997; 56: 463-469.

Chang K, Yang SM, Kim SH, et al. Smoking and rheumatoid arthritis. Int J Mol Sci 2014; 15: 22279-22295.

Vessey MP, Villard-Mackintosh L, Yeates D. Oral contraceptives, cigarette smoking and other factors in relation to arthritis. Contraception 1987; 35: 457-464.

Criswell LA, Merlino LA, Cerhan JR, et al. Cigarette smoking and the risk of rheumatoid arthritis among postmenopausal women. Am J Med 2002; 112: 465-471.

Di Giuseppe D, Discacciati A, Orsini N, et al. Cigarette smoking and risk of rheumatoid arthritis: a dose-response meta-analysis. Arthritis Res Ther 2014; 16: R61.

9 Jónsson T, Thorsteinsson J, Valdimarsson H. Does smoking stimulate rheumatoid factor production in non-rheumatic individuals? APMIS 1998; 106: 970-974.

Haye Salinas MJ, Retamozo S, Alvarez AC, et al. Effects of cigarette smoking on early arthritis: a cross-sectional study - data from the Argentine Consortium for Early Arthritis (CONAART). Rheumatol Int 2015; 35: 855-859. 
Makrygiannakis D, Hermansson M, Ulfgren A-K, et al. Smoking increases peptidylarginine deiminase 2 enzyme expression in human lungs and increases citrullination in BAL cells. Ann Rheum Dis 2008; 67: 1488-1492.

Klareskog L, Stolt P, Lundberg K, et al. A new model for an etiology of rheumatoid arthritis: smoking may trigger HLA-DR (shared epitope)-restricted immune reactions to autoantigens modified by citrullination. Arthritis Rheum 2006; 54: 38-46.

Karlson EW, Chang S-C, Cui J, et al. Gene-environment interaction between HLA-DRB1 shared epitope and heavy cigarette smoking in predicting incident rheumatoid arthritis. Ann Rheum Dis 2010; 69: 54-60.

Inoue $\mathrm{Y}$, Nakajima A, Tanaka E, et al. Effect of smoking on remission proportions differs between male and female patients with rheumatoid arthritis: a study based on the IORRA survey. $J$ Rheumatol 2015; 42: 1083-1089.

Krol A, Garred P, Heegaard NHH, et al. Interactions between smoking, increased serum levels of anti-CCP antibodies, rheumatoid factors, and erosive joint disease in patients with early, untreated rheumatoid arthritis. Scand J Rheumatol 2015; 44: 8-12.

Spagnolo P, Grunewald J, du Bois RM. Genetic determinants of pulmonary fibrosis: evolving concepts. Lancet Respir Med 2014; 2: 416-428.

de Lauretis A, Veeraraghavan S, Renzoni E. Review series: aspects of interstitial lung disease: connective tissue disease-associated interstitial lung disease: how does it differ from IPF? How should the clinical approach differ? Chron Respir Dis 2011; 8: 53-82.

Cavagna L, Monti S, Grosso V, et al. The multifaceted aspects of interstitial lung disease in rheumatoid arthritis. BioMed Res Int 2013; 2013: 759760.

Bongartz T, Nannini C, Medina-Velasquez YF, et al. Incidence and mortality of interstitial lung disease in rheumatoid arthritis: a population-based study. Arthritis Rheum 2010; 62: 1583-1591.

Mori S, Koga Y, Sugimoto M. Different risk factors between interstitial lung disease and airway disease in rheumatoid arthritis. Respir Med 2012; 106: 1591-1599.

Gochuico BR, Avila NA, Chow CK, et al. Progressive preclinical interstitial lung disease in rheumatoid arthritis. Arch Intern Med 2008; 168: 159-166.

Doyle TJ, Patel AS, Hatabu $\mathrm{H}$, et al. Detection of rheumatoid arthritis-interstitial lung disease is enhanced by serum biomarkers. Am J Respir Crit Care Med 2015; 191: 1403-1412.

Bendstrup E, Møller J, Kronborg-White S, et al. Interstitial lung disease in rheumatoid arthritis remains a challenge for clinicians. J Clin Med. 2019; 8: 2038.

Raghu G, Remy-Jardin M, Myers JL, et al. Diagnosis of idiopathic pulmonary fibrosis. An official ATS/ERS/ JRS/ALAT clinical practice guideline. Am J Respir Crit Care Med 2018; 198: e44-e68.

Travis WD, Costabel U, Hansell DM, et al. An official American Thoracic Society/European Respiratory Society statement: update of the international multidisciplinary classification of the idiopathic interstitial pneumonias. Am J Respir Crit Care Med 2013; 188: 733-748.

Antoniou KM, Walsh SL, Hansell DM, et al. Smoking-related emphysema is associated with idiopathic pulmonary fibrosis and rheumatoid lung. Respirology 2013; 18: 1191-1196.

Garcia JG, Parhami N, Killam D, et al. Bronchoalveolar lavage fluid evaluation in rheumatoid arthritis. Am Rev Respir Dis 1986; 133: 450-454.

Flaherty KR, Thwaite EL, Kazerooni EA, et al. Radiological versus histological diagnosis in UIP and NSIP: survival implications. Thorax 2003; 58: 143-148.

Kim EJ, Elicker BM, Maldonado F, et al. Usual interstitial pneumonia in rheumatoid arthritis-associated interstitial lung disease. Eur Respir J 2010; 35: 1322-1328.

Shaw M, Collins BF, Ho LA, et al. Rheumatoid arthritis-associated lung disease. Eur Respir Rev 2015; 24: 1-16. Lee H-K, Kim DS, Yoo B, et al. Histopathologic pattern and clinical features of rheumatoid arthritis-associated interstitial lung disease. Chest 2005; 127: 2019-2027.

Assayag D, Elicker BM, Urbania TH, et al. Rheumatoid arthritis-associated interstitial lung disease: radiologic identification of usual interstitial pneumonia pattern. Radiology 2014; 270: 583-588.

Nakamura Y, Suda T, Kaida Y, et al. Rheumatoid lung disease: prognostic analysis of 54 biopsy-proven cases. Respir Med 2012; 106: 1164-1169.

4 Turesson C, Matteson EL, Colby TV, et al. Increased CD4+ T cell infiltrates in rheumatoid arthritis-associated interstitial pneumonitis compared with idiopathic interstitial pneumonitis. Arthritis Rheum 2005; 52: 73-79.

Assayag D, Lubin M, Lee JS, et al. Predictors of mortality in rheumatoid arthritis-related interstitial lung disease. Respirology 2014; 19: 493-500.

Solomon JJ, Chung JH, Cosgrove GP, et al. Predictors of mortality in rheumatoid arthritis-associated interstitial lung disease. Eur Respir J 2016; 47: 588-596.

Wang D, Zhang J, Lau J, et al. Mechanisms of lung disease development in rheumatoid arthritis. Nat Rev Rheumatol 2019; 15: 581-596.

Tsuchiya Y, Takayanagi N, Sugiura H, et al. Lung diseases directly associated with rheumatoid arthritis and their relationship to outcome. Eur Respir J 2011; 37: 1411-1417. 
Nurmi HM, Purokivi MK, Kärkkäinen MS, et al. Variable course of disease of rheumatoid arthritis-associated usual interstitial pneumonia compared to other subtypes. BMC Pulm Med 2016; 16: 107.

Yunt ZX, Chung JH, Hobbs S, et al. High resolution computed tomography pattern of usual interstitial pneumonia in rheumatoid arthritis-associated interstitial lung disease: relationship to survival. Respir Med 2017; 126: 100-104.

Buttgereit F, Straub RH, Wehling M, et al. Glucocorticoids in the treatment of rheumatic diseases: an update on the mechanisms of action. Arthritis Rheum 2004; 50: 3408-3417.

Ortiz LA, Lasky J, Hamilton RF, et al. Expression of TNF and the necessity of TNF receptors in bleomycin-induced lung injury in mice. Exp Lung Res 1998; 24: 721-743.

Derksen VFAM, Huizinga TWJ, van der Woude D. The role of autoantibodies in the pathophysiology of rheumatoid arthritis. Semin Immunopathol 2017; 39: 437-446.

Scott DL, Wolfe F, Huizinga TWJ. Rheumatoid arthritis. Lancet 2010; 376: 1094-1108.

Valesini G, Gerardi MC, lannuccelli C, et al. Citrullination and autoimmunity. Autoimmun Rev 2015; 14 490-497.

Rantapää-Dahlqvist S, de Jong BAW, Berglin E, et al. Antibodies against cyclic citrullinated peptide and IgA rheumatoid factor predict the development of rheumatoid arthritis. Arthritis Rheum 2003; 48: 2741-2749.

Nishimura K, Sugiyama D, Kogata Y, et al. Meta-analysis: diagnostic accuracy of anti-cyclic citrullinated peptide antibody and rheumatoid factor for rheumatoid arthritis. Ann Intern Med 2007; 146: 797-808.

Whiting PF, Smidt N, Sterne JAC, et al. Systematic review: accuracy of anti-citrullinated peptide antibodies for diagnosing rheumatoid arthritis. Ann Intern Med 2010; 152: 456-464.

Lee DM, Schur PH. Clinical utility of the anti-CCP assay in patients with rheumatic diseases. Ann Rheum Dis 2003; 62: 870-874.

Aletaha D, Neogi T, Silman AJ, et al. 2010 Rheumatoid arthritis classification criteria: an American College of Rheumatology/European League Against Rheumatism collaborative initiative. Arthritis Rheum 2010; 62: 2569-2581.

Holers VM, Demoruelle MK, Kuhn KA, et al. Rheumatoid arthritis and the mucosal origins hypothesis: protection turns to destruction. Nat Rev Rheumatol 2018; 14: 542-557.

Scher JU, Joshua V, Artacho A, et al. The lung microbiota in early rheumatoid arthritis and autoimmunity. Microbiome 2016; 4: 60.

Paulin F, Doyle TJ, Fletcher EA, et al. Rheumatoid arthritis-associated interstitial lung disease and idiopathic pulmonary fibrosis: shared mechanistic and phenotypic traits suggest overlapping disease mechanisms. Rev Invest Clin 2015; 67: 280-286.

Reynisdottir G, Olsen H, Joshua V, et al. Signs of immune activation and local inflammation are present in the bronchial tissue of patients with untreated early rheumatoid arthritis. Ann Rheum Dis 2016; 75: 1722-1727.

Zhang J, Wang D, Wang L, et al. Profibrotic effect of IL-17A and elevated IL-17RA in idiopathic pulmonary fibrosis and rheumatoid arthritis-associated lung disease support a direct role for IL-17A/IL-17RA in human fibrotic interstitial lung disease. Am J Physiol Lung Cell Mol Physiol 2019; 316: L487-L497.

Broekelmann TJ, Limper AH, Colby TV, et al. Transforming growth factor beta 1 is present at sites of extracellular matrix gene expression in human pulmonary fibrosis. Proc Natl Acad Sci USA 1991; 88: 6642-6646.

Rangel-Moreno J, Hartson L, Navarro C, et al. Inducible bronchus-associated lymphoid tissue (iBALT) in patients with pulmonary complications of rheumatoid arthritis. J Clin Invest 2006; 116: 3183-3194.

Xiong L, Xiong L, Ye $\mathrm{H}$, et al. Animal models of rheumatoid arthritis-associated interstitial lung disease. Immun Inflamm Dis 2021; 9: 37-47.

Wu EK, Ambrosini RD, Kottmann RM, et al. Reinterpreting evidence of rheumatoid arthritis-associated interstitial lung disease to understand etiology. Curr Rheumatol Rev 2019; 15: 277-289.

Hallowell RW, Horton MR. Interstitial lung disease in patients with rheumatoid arthritis: spontaneous and drug induced. Drugs 2014; 74: 443-450.

Song JW, Lee H-K, Lee CK, et al. Clinical course and outcome of rheumatoid arthritis-related usual interstitial pneumonia. Sarcoidosis Vasc Diffuse Lung Dis 2013; 30: 103-112.

Yamano $\mathrm{Y}$, Taniguchi $\mathrm{H}$, Kondoh $\mathrm{Y}$, et al. Multidimensional improvement in connective tissue disease-associated interstitial lung disease: two courses of pulse dose methylprednisolone followed by low-dose prednisone and tacrolimus. Respirology 2018; 23: 1041-1048.

Idiopathic Pulmonary Fibrosis Clinical Research Network, Martinez FJ, de Andrade JA, et al. Randomized trial of acetylcysteine in idiopathic pulmonary fibrosis. N Engl J Med 2014; 370: 2093-2101.

Li L, Liu R, Zhang Y, et al. A retrospective study on the predictive implications of clinical characteristics and therapeutic management in patients with rheumatoid arthritis-associated interstitial lung disease. Clin Rheumatol 2020; 39: 1457-1470. 

2014; 73: 492-509.

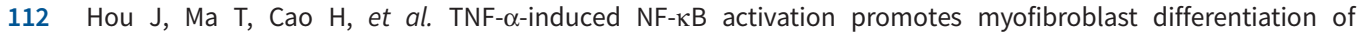
LR-MSCs and exacerbates bleomycin-induced pulmonary fibrosis. J Cell Physiol 2018; 233: 2409-2419.

113 Cassone G, Manfredi A, Vacchi C, et al. Treatment of rheumatoid arthritis-associated interstitial lung disease: lights and shadows. J Clin Med 2020; 9: 1082.

114 Ramos-Casals M, Perez-Alvarez R, Perez-de-Lis M, et al. Pulmonary disorders induced by monoclonal antibodies in patients with rheumatologic autoimmune diseases. Am J Med 2011; 124: 386-394.

115 Dixon WG, Hyrich KL, Watson KD, et al. Influence of anti-TNF therapy on mortality in patients with rheumatoid arthritis-associated interstitial lung disease: results from the British Society for Rheumatology Biologics Register. Ann Rheum Dis 2010; 69: 1086-1091.

116 Detorakis EE, Magkanas E, Lasithiotaki I, et al. Evolution of imaging findings, laboratory and functional parameters in rheumatoid arthritis patients after one year of treatment with anti-TNF- $\alpha$ agents. Clin Exp Rheumatol 2017; 35: 43-52.

117 Herrinton LJ, Harrold LR, Liu L, et al. Association between anti-TNF- $\alpha$ therapy and interstitial lung disease. Pharmacoepidemiol Drug Saf 2013; 22: 394-402.

118 Perez-Alvarez R, Perez-de-Lis M, Diaz-Lagares C, et al. Interstitial lung disease induced or exacerbated by TNF-targeted therapies: analysis of 122 cases. Semin Arthritis Rheum 2011; 41: 256-264.

119 Raghu G, Brown KK, Costabel U, et al. Treatment of idiopathic pulmonary fibrosis with etanercept: an exploratory, placebo-controlled trial. Am J Respir Crit Care Med 2008; 178: 948-955.

120 Flaherty KR, Wells AU, Cottin V, et al. Nintedanib in progressive fibrosing interstitial lung diseases. $N$ Engl $J$ Med 2019; 381: 1718-1727.

121 Wells $\mathrm{AU}$, Flaherty KR, Brown KK, et al. Nintedanib in patients with progressive fibrosing interstitial lung diseases - subgroup analyses by interstitial lung disease diagnosis in the INBUILD trial: a randomised, double-blind, placebo-controlled, parallel-group trial. Lancet Respir Med 2020; 8: 453-460. 
122 Schaefer CJ, Ruhrmund DW, Pan L, et al. Antifibrotic activities of pirfenidone in animal models. Eur Respir Rev 2011; 20: 85-97.

123 Wu C, Lin $\mathrm{H}$, Zhang X. Inhibitory effects of pirfenidone on fibroblast to myofibroblast transition in rheumatoid arthritis-associated interstitial lung disease via the downregulation of activating transcription factor 3 (ATF3). Int Immunopharmacol 2019; 74: 105700.

124 Maher TM, Corte TJ, Fischer A, et al. Pirfenidone in patients with unclassifiable progressive fibrosing interstitial lung disease: a double-blind, randomised, placebo-controlled, phase 2 trial. Lancet Respir Med 2020; 8: 147-157.

125 Behr J, Neuser P, Prasse A, et al. Exploring efficacy and safety of oral pirfenidone for progressive, non-IPF lung fibrosis (RELIEF) - a randomized, double-blind, placebo-controlled, parallel group, multi-center, phase II trial. BMC Pulm Med 2017; 17: 122.

126 George PM, Spagnolo P, Kreuter M, et al. Progressive fibrosing interstitial lung disease: clinical uncertainties, consensus recommendations, and research priorities. Lancet Respir Med 2020; 8: 925-934.

127 Solomon DH, Karlson EW, Rimm EB, et al. Cardiovascular morbidity and mortality in women diagnosed with rheumatoid arthritis. Circulation 2003; 107: 1303-1307.

128 Sparks JA, Chang S-C, Liao KP, et al. Rheumatoid arthritis and mortality among women during 36 years of prospective follow-up: results from the nurses' health study. Arthritis Care Res 2016; 68: 753-762.

129 Jeganathan N, Nguyen E, Sathananthan M. Rheumatoid arthritis and associated-interstitial lung disease: mortality rates and trends. Ann Am Thorac Soc 2021; in press [https://doi.org/10.1513/AnnalsATS. 202102-1150C].

130 Geddes DM, Webley M, Emerson PA. Airways obstruction in rheumatoid arthritis. Ann Rheum Dis 1979; 38: 222-225.

131 Hassan WU, Keaney NP, Holland CD, et al. Bronchial reactivity and airflow obstruction in rheumatoid arthritis. Ann Rheum Dis 1994; 53: 511-514.

132 Yunt ZX, Solomon JJ. Lung disease in rheumatoid arthritis. Rheum Dis Clin North Am 2015; 41: 225-236.

133 Wilczynska MM, Condliffe AM, McKeon DJ. Coexistence of bronchiectasis and rheumatoid arthritis: revisited. Respir Care 2013; 58: 694-701.

134 Geri G, Dadoun S, Bui T, et al. Risk of infections in bronchiectasis during disease-modifying treatment and biologics for rheumatic diseases. BMC Infect Dis 2011; 11: 304.

135 Hayakawa H, Sato A, Imokawa S, et al. Bronchiolar disease in rheumatoid arthritis. Am J Respir Crit Care Med 1996; 154: 1531-1536.

136 Lynch JP, Weigt SS, DerHovanessian A, et al. Obliterative (constrictive) bronchiolitis. Semin Respir Crit Care Med 2012; 33: 509-532.

137 Lin E, Limper AH, Moua T. Obliterative bronchiolitis associated with rheumatoid arthritis: analysis of a single-center case series. BMC Pulm Med 2018; 18: 105.

138 Fernández Pérez ER, Krishnamoorthy M, Brown KK, et al. FEV1 over time in patients with connective tissue disease-related bronchiolitis. Respir Med 2013; 107: 883-889.

139 Devouassoux G, Cottin V, Liote $\mathrm{H}$, et al. Characterisation of severe obliterative bronchiolitis in rheumatoid arthritis. Eur Respir J 2009; 33: 1053-1061.

140 Cortot $\mathrm{AB}$, Cottin V, Miossec $\mathrm{P}$, et al. Improvement of refractory rheumatoid arthritis-associated constrictive bronchiolitis with etanercept. Respir Med 2005; 99: 511-514.

141 Yadav H, Peters SG, Keogh KA, et al. Azithromycin for the treatment of obliterative bronchiolitis after hematopoietic stem cell transplantation: a systematic review and meta-analysis. Biol Blood Marrow Transplant 2016; 22: 2264-2269.

142 Fischer A, Strek ME, Cottin V, et al. Proceedings of the American College of Rheumatology/Association of Physicians of Great Britain and Ireland Connective Tissue Disease-Associated Interstitial Lung Disease Summit: a multidisciplinary approach to address challenges and opportunities. Arthritis Rheumatol 2019; 71: 182-195. 